\title{
Prognostic and Predictive Implications of PTEN in Breast Cancer: Unfulfilled Promises but Intriguing Perspectives
}

\author{
Luisa Carbognin $1,2,+$, Federica Miglietta ${ }^{3,4,+}$, Ida Paris ${ }^{2}$ and Maria Vittoria Dieci ${ }^{3,4, *(D)}$ \\ 1 Department of Medicine, University of Verona, 37126 Verona, Italy; luisa.carbognin@univr.it \\ 2 Division of Gynecologic Oncology, Department of Woman and Child Health and Public Health, Fondazione \\ Policlinico Universitario A. Gemelli IRCCS, 00168 Roma, Italy; ida.paris@policlinicogemelli.it \\ 3 Department of Surgery, Oncology and Gastroenterology, University of Padova, 35128 Padova, Italy; \\ federica.miglietta@iov.veneto.it \\ 4 Division of Medical Oncology 2, Istituto Oncologico Veneto IRCCS, 35128 Padova, Italy \\ * Correspondence: mariavittoria.dieci@unipd.it; Tel.: +39-049-821-5295; Fax: +39-049-821-5932 \\ $+\quad$ L.C. and F.M. share the first co-authorship.
}

Received: 3 August 2019; Accepted: 15 September 2019; Published: 19 September 2019

\begin{abstract}
The characterization of tumor biology and consequently the identification of prognostic and predictive biomarkers represent key issues for the translational research in breast cancer (BC). Phosphatase and tensin homolog deleted on chromosome ten (PTEN), the negative regulator of the proto-oncogenic phosphatidylinositol-3-kinase (PI3K)/protein kinase B (Akt) pathway, constitutes one of the most intriguing tumor suppressor genes involved in a series of biological processes, such as cell growth and survival, cellular migration and genomic stability. Loss of PTEN activity, due to protein, genetic or epigenetic alterations, was reported in up to almost half of $\mathrm{BC}$ cases. Recently, besides the role of PTEN in the pathogenesis of BC, investigated for over 20 years after the PTEN discovery, several retrospective and prospective translational studies, in the early and advanced setting, reported controversial results regarding the association between PTEN functional status and both clinical outcome and response to various BC treatments. This review explores the pre-clinical and clinical role of PTEN in BC with regard to the potential association of PTEN with prognosis and treatment response or resistance, underlying the complexity of the interpretation of available results and suggesting potential future perspectives.
\end{abstract}

Keywords: PTEN; breast cancer; prognosis; predictive role; treatment response; survival

\section{Introduction}

In the recent era of precision medicine, translational and clinical research have been matched together with enormous efforts in order to characterize tumors and customize treatment according to genetic, epigenetic or proteomic alterations. In the context of breast cancer (BC), the integration between technology, innovation and translational research represents a key issue to finally understand tumor biology and consequently to identify potential biomarkers of drug response or resistance. In this regard, phosphatase and tensin homolog deleted on chromosome ten (PTEN) represents one of the most frequently altered genes in human cancer, including BC. The role of PTEN as a key tumor suppressor gene, implicated in cell cycle progression, cell growth and survival in multiple tumor types, was discovered in 1997 by three independent research teams [1-3]. Specifically, PTEN encodes for dual-specificity lipid and protein phosphatase which downregulates the phosphatidylinositol-3-kinase $(\mathrm{PI} K \mathrm{~K}) /$ protein kinase B (Akt) signaling, a transduction pathway involved in a series of biological processes such as cellular motility, invasion, proliferation and survival. In particular, PTEN 
dephosphorylates the 3'-group of the phosphatidylinositol (3,4,5)-trisphosphate (PIP3), a pleiotropic second messenger that activates downstream signaling components, such as the protein kinase Akt, which stimulates downstream signaling pathways required for pro-survival processes. Thus, the dephosphorylation of PIP3 by PTEN turns off the PI3K/Akt pathway, inhibiting the growth and survival signals $[4,5]$. Moreover, a series of phosphatase-independent biological actions of PTEN in the cytoplasm and the nucleus have been described. These mechanisms include the binding of TP53, increasing TP53 stability and transcriptional activity, and the interaction with the APC/C (anaphase-promoting complex/cyclosome), enhancing the tumor-suppressive activity of the APC-CDH1 complex [6,7].

PTEN is also implicated in DNA repair and genome stability. Interestingly, it has been recently reported that in response to DNA damage, PTEN is phosphorylated on Tyr240, binds to chromatin and facilitates the recruitment of RAD51 to promote DNA repair, and thus, resistance to ionizing radiation therapy [8].

The loss of PTEN activity, identified in a series of primary and metastatic tumors such as breast cancer, leads into uncontrolled transduction of the PI3K signal. The PTEN inactivation is mainly due to somatic mutations including missense and nonsense mutations, monoallelic or biallelic deletion of PTEN gene locus, epigenetic silencing through promoter methylation, PTEN protein degradation and post-translational alterations of PTEN protein [9]. In particular, the post-translational modifications of wild-type PTEN (e.g., phosphorylation, mono- or poly-ubiquitination, sumoylation, oxidation and acetylation) are known to tightly regulate the function of the tumor suppressor as the result of altered subcellular localization, protein/protein interaction and/or phosphatase activity, independently of PTEN accumulation [10].

This process might explain some discrepancies related to PTEN expression levels, evaluated by next-generation sequencing or immunohistochemistry, and its biological functions.

Moreover, germline PTEN mutations are detected in inherited cancer syndromes, such as the Cowden syndrome characterized by the presence of multiple hamartomas and an increased risk for breast, thyroid, endometrium, kidney and colorectum malignancies [11].

Loss of heterozygosity at the PTEN locus was reported in nearly $40 \%-50 \%$ of breast tumors, whereas the loss of PTEN function due to PTEN mutations was detected in $5 \%-10 \%$ of BC cases, with frameshift representing the most frequent mechanism [12,13]. As for other solid tumors, epigenetic mechanisms of PTEN modulation have also been reported for BC $[3,14]$. Mutations in the PIK3CA gene represent another frequent mechanism of dysregulation of the PI3k/Akt pathway. Despite the fact that PTEN loss and PIK3CA mutations are commonly considered mutually exclusive events, a series of data reported concomitant alterations of the two genes in BC $[15,16]$.

Although a large series of key data unravelling the role of PTEN in the pathogenesis of (solid) tumors have been currently reported to date, its contribution to BC progression in patients and its role as a potential predictor of resistance/efficacy to targeted agents present controversial issues and still require further investigation.

\section{Preclinical Studies of Chromosome Ten's (PTEN) Role in Breast Cancer (BC)}

In the specific context of BC susceptibility, the role of PTEN was explored in PTEN-hypermorphic mouse models, expressing $80 \%$ of the normal levels of PTEN. In these mice, the slight decrease in PTEN expression seems to be sufficient to promote BC development and progression [17].

Several post-transcriptional mechanisms, including microRNA (miRNA) regulation, control PTEN expression. Recently, the RNA-binding motif 38 (RBM38), a RNA-binding protein and a target of the p53 family, was shown to regulate the PTEN expression in BC cells and tissues through its transcript stability. Thus, RBM38 may induce growth suppression by enhancing PTEN expression [18]. Moreover, a series of reports have pointed out the role of PTEN expression levels in stromal cells in a mouse model of BC. The PTEN genetic inactivation in stromal fibroblasts of mouse mammary glands may enhance the initiation and cancerous transformation of mammary epithelial tumors [19]. 
Besides the role of miRNAs in cell proliferation, a series of studies reported that certain specific miRNAs can be involved in drug-resistance [20]. In MCF-7 BC cells lines, the miRNA-222 may promote resistance to adriamycin through modulation of the PTEN/Akt/FOXO1 pathway [21].

With regard to targeted agents, pre-clinical data suggested a role of PTEN in treatment resistance to Trastuzumab. In HER2-positive (HER2+) BC cell-lines and xenografts, PTEN deficiency seems to contribute to Trastuzumab resistance by dephosphorylation and activation of SRC proto-oncogene nonreceptor tyrosine-protein kinase. Indeed, SRC seems to be hyperactivated in several Trastuzumab resistance models. Moreover, SRC activation and PTEN deficiency may be associated with poor outcomes in patients who received Trastuzumab-based therapy [14,22]. Thus, PTEN deficiency represents a potential predictor for Trastuzumab resistance.

Recently, a series of PI3K inhibitors has been investigated in BC pre-clinical and clinical settings. In pre-clinical studies, the predictive role of PTEN to PI3K inhibitors remains controversial. In this context, Tanaka et al. showed that PTEN deficiency was not associated with a response to CH5132799 (a class I PI3K inhibitor) in cell-lines and xenografts, including BC models. Conversely, PIK3CA mutation was suggested to be a predictor of a response [23]. A similar predictive role for PIK3CA mutation and not for PTEN loss of function was found in an in vitro study testing NVP-BEZ235 (a dual PI3K/mTOR inhibitor) [24]. In contrast, the analysis conducted by Stemke-Hale et al., suggested that PTEN loss in 12 hormone receptor-positive (HR+) BC cell lines was more predictive of response to LY294002-a potent PI3K inhibitor-than PIK3CA mutations [15]. Finally, Juric et al., reported that PTEN down-regulation was associated with resistance to the PI3K p110-alpha selective inhibitor BYL719, but not to the pan-PI3K inhibitor BKM120 in BC cell lines [25].

Interestingly, it has been suggested that PTEN loss may affect immune checkpoint regulation. Mittendorf et al., reported that PTEN knockdown increased cell-surface programmed cell death ligand 1 (PD-L1) expression and PD-L1 transcript in triple-negative BC cell lines, ultimately resulting, at the functional level, in decreased T-cell proliferation and increased T-cell apoptosis, as suggested by co-culture experiments [26].

\section{Clinical Studies of PTEN's Role in BC}

Despite its potential biological relevance, the clinical utility of this biomarker remains controversial. Here, we reviewed available evidence on the prognostic and/or predictive role of PTEN status in BC patients, derived from biomarker analyses of patients enrolled in clinical trials.

\subsection{Association with Prognosis}

Although most studies failed to report an association between PTEN loss and prognosis in BC patients enrolled in clinical trials, emerging evidence suggests that lack or decrease in PTEN expression may be associated with worse outcomes in HR+/HER2 negative (HER2-) or HER2+ BC.

In detail, results from a biomarker analysis of 764 samples from the Breast International Group 1-98 (which randomized post-menopausal HR+ early BC patients to receive in the adjuvant setting tamoxifen, letrozole or the sequential treatment with letrozole followed by tamoxifen) revealed a non-significant trend towards increased risk of distant recurrence for patients with tumors harboring a PTEN mutation by next-generation sequencing, as compared with patients with wild-type PTEN (HR $1.67,95 \%$ CI $0.69-4.06, p=0.26$ ) [27].

With regard to HER2+ BC, several randomized phase III trials did not report any significant association between survival and PTEN status assessed at protein level expression, either in the early [28,29] or advanced setting [30-32].

On the other hand, several authors have recently suggested that PTEN status may actually retain a prognostic role in this $\mathrm{BC}$ subtype, as highlighted by translational analyses from various prospective BC cohorts. Notably, Stern et al., reported that HER2+ BC patients treated with chemotherapy with or without Trastuzumab in the context of the adjuvant phase III BCIRG 006 trial who lacked PTEN 
expression exhibited poorer disease-free survival (DFS) and overall survival (OS), as compared to those with detectable PTEN staining by immunohistochemistry [33].

DNA analysis from the phase III APHINITY trial, randomizing patients with HER2+ early BC to receive adjuvant chemotherapy plus Trastuzumab and Pertuzumab versus chemotherapy plus Trastuzumab and placebo, revealed that PI3K/Akt/PTEN alterations (comprising PTEN homozygous deletion or any PTEN alteration) predicted worse outcome in terms of invasive DFS in a pooled analysis of both treatment arms [34].

\subsection{Association with Treatment Response}

Besides its potential prognostic role, PTEN status has also been suggested as a predictor of the response to different antitumor agents as revealed by translational analyses of several phase II and phase III clinical trials in HER2- (Table 1) and HER2+ BC (Table 2). 
Table 1. Phase II and phase III clinical trials with translational analyses exploring the predictive role of chromosome ten (PTEN) in human epidermal growth factor receptor 2 (HER2)-negative breast cancer.

\begin{tabular}{|c|c|c|c|c|c|c|c|c|c|}
\hline Drug Family & Agent & Trial & Phase & Population & Setting & Arms & $\begin{array}{c}\text { N pts } \\
\text { (Translational } \\
\text { Analysis) } \\
\end{array}$ & PTEN Analysis & Main Results \\
\hline \multirow[t]{3}{*}{$\begin{array}{l}\text { mTOR } \\
\text { Inhibitor }\end{array}$} & \multirow[t]{3}{*}{ Everolimus } & TAMRAD [35] & II & $\begin{array}{l}\text { HR+/HER2- } \\
\text { MBC }\end{array}$ & AI-resistant & $\begin{array}{c}\text { Tamoxifene }+ \\
\text { Everolimus } \\
\text { Tamoxifene }\end{array}$ & $\begin{array}{l}30 \\
25\end{array}$ & $\begin{array}{c}\text { IHC clone 138G6 Cell } \\
\text { Signaling Technology ( } \% \text { and } \\
\text { intensity: } \\
1+, 2+, 3+)\end{array}$ & No predictive role (TTP) \\
\hline & & \multirow{2}{*}{$\begin{array}{c}\text { BOLERO-2 } \\
\text { [36] }\end{array}$} & \multirow[b]{2}{*}{ III } & \multirow{2}{*}{$\begin{array}{l}\text { HR+/HER2- } \\
\text { MBC }\end{array}$} & \multirow[b]{2}{*}{ AI-resistant } & \multirow{2}{*}{$\begin{array}{c}\text { Exemestane + } \\
\text { Everolimus } \\
\text { Exemestane + } \\
\text { Placebo } \\
\end{array}$} & \multirow{2}{*}{$\begin{array}{l}209 \\
93\end{array}$} & NGS (at least 1 mutation) & No predictive role (PFS) \\
\hline & & & & & & & & $\begin{array}{c}\text { IHC (low-PTEN: H-score < } \\
10 \text { ) }\end{array}$ & No predictive role (PFS) \\
\hline \multirow{6}{*}{$\begin{array}{c}\text { AKT } \\
\text { Inhibitor }\end{array}$} & \multirow{4}{*}{ Ipatasertib } & \multirow[b]{2}{*}{ LOTUS [37] } & \multirow[b]{2}{*}{ II } & \multirow[b]{2}{*}{$\begin{array}{l}\mathrm{TN} \\
\mathrm{MBC}\end{array}$} & \multirow[b]{2}{*}{ First Line } & \multirow[b]{2}{*}{$\begin{array}{c}\text { Paclitaxel + } \\
\text { Ipatasertib } \\
\text { Paclitaxel + Placebo }\end{array}$} & \multirow[b]{2}{*}{$\begin{array}{c}54 \\
47 \text { (IHC) } \\
49 \text { (NGS) }\end{array}$} & $\begin{array}{l}\text { IHC (low PTEN score } 0 \text { in at } \\
\text { least } 50 \% \text { of tumor cells) }\end{array}$ & No predictive role (PFS) \\
\hline & & & & & & & & $\begin{array}{l}\text { NGS (genetic-inactivating } \\
\text { alterations) }\end{array}$ & $\begin{array}{c}\mathrm{mPFS} \text { of } 9.0 \mathrm{~m} \text { with ipatasertib } \\
\text { versus } 4.9 \mathrm{~m} \text { with placebo } \\
\text { (non-stratified HR } 0.44, p= \\
\text { 0.041) in PIK3CA/AKT1/ } \\
\text { PTEN-altered subgroup }\end{array}$ \\
\hline & & \multirow{2}{*}{$\begin{array}{l}\text { FARLAINE } \\
{[38]}\end{array}$} & \multirow[b]{2}{*}{ II } & \multirow{2}{*}{$\begin{array}{l}\text { TN } \\
\text { EBC }\end{array}$} & \multirow[b]{2}{*}{ Neoadjuvant } & \multirow{2}{*}{$\begin{array}{c}\text { CT + Ipatasertib } \\
\text { CT + Placebo }\end{array}$} & \multirow{2}{*}{$\begin{array}{l}\text { Overall } \\
132\end{array}$} & $\begin{array}{l}\text { IHC clone Ventana SP218 } \\
\text { (low PTEN: score } 0 \%-50 \% \text { of } \\
\text { tumor cells) }\end{array}$ & $\begin{array}{l}\text { pCR rate } 16 \% \text { with ipatasertib } \\
\text { versus } 13 \% \text { with placebo in } \\
\text { PTEN-low population }\end{array}$ \\
\hline & & & & & & & & $\begin{array}{l}\text { NGS (PTEN genomic } \\
\text { alterations) }\end{array}$ & $\begin{array}{l}\text { pCR rate } 18 \% \text { with ipatasertib } \\
\text { versus } 12 \% \text { with placebo in } \\
\text { PIK3CA/AKT1/PTEN-altered } \\
\text { subgroup }\end{array}$ \\
\hline & \multirow{2}{*}{ Capivasertib } & PAKT [39] & II & $\begin{array}{l}\mathrm{TN} \\
\mathrm{MBC}\end{array}$ & First Line & $\begin{array}{c}\text { Paclitaxel + } \\
\text { Capivasertib } \\
\text { Paclitaxel + Placebo }\end{array}$ & Overall 140 & NGS (genetic alteration) & $\begin{array}{c}\text { mPFS of } 9.3 \mathrm{~m} \text { with } \\
\text { capivasertib versus } 3.7 \mathrm{~m} \text { with } \\
\text { placebo in PIK3CA, AKT1 or } \\
\text { PTEN-altered subgroup (HR } \\
0.30, p=0.01 \text { ) }\end{array}$ \\
\hline & & FAKTION [40] & II & $\begin{array}{l}\text { HR+/HER2- } \\
\text { MBC }\end{array}$ & AI-resistant & $\begin{array}{c}\text { Fulvestrant }+ \\
\text { Capivasertib } \\
\text { Fulvestrant }+ \text { Placebo }\end{array}$ & $\begin{array}{l}69 \\
71\end{array}$ & $\mathrm{IHC}$ & No predictive role (PFS) \\
\hline
\end{tabular}


Table 1. Cont.

\begin{tabular}{|c|c|c|c|c|c|c|c|c|c|}
\hline Drug Family & Agent & Trial & Phase & Population & Setting & Arms & $\begin{array}{c}\text { N pts } \\
\text { (Translational } \\
\text { Analysis) }\end{array}$ & PTEN Analysis & Main Results \\
\hline $\begin{array}{c}\text { PARP } \\
\text { Inhibitor }\end{array}$ & Talazoparib & $\begin{array}{c}\text { NCT02401347 } \\
{[41]}\end{array}$ & II & $\begin{array}{l}\text { HER2- } \\
\text { MBC (plus } \\
\text { other solid } \\
\text { tumor) }\end{array}$ & Pretreated & Talazoparib & $\begin{array}{l}20(13 \\
\text { BRCA1/2 wt } \\
\text { MBC and } 7 \\
\text { non- breast })\end{array}$ & $\begin{array}{l}\text { NGS (homologous } \\
\text { recombination pathway: } \\
\text { PTEN gene mutation) }\end{array}$ & $\begin{array}{l}3 \text { pts had a RECIST response } \\
\text { (ORR }=25 \%, 2 \text { gPALB2, } 1 \\
\text { gCHEK2/gFANCA/sPTEN) } \\
\text { and } 3 \text { additional pts (gPALB2, } \\
\text { sATR, sPTEN) had SD } \geq 6 \text { m. } \\
(\text { CBR }=50 \%)\end{array}$ \\
\hline $\begin{array}{l}\text { Endocrine } \\
\text { Therapy }\end{array}$ & $\begin{array}{l}\text { Anastrozole/ } \\
\text { Fulvestrant }\end{array}$ & $\begin{array}{c}\text { CARMINA } 02 \\
\text { [42] }\end{array}$ & II & $\begin{array}{l}\text { HER2- } \\
\text { EBC }\end{array}$ & Neoadjuvant & $\begin{array}{c}\text { Anastrozole } \\
\text { Fulvestrant }\end{array}$ & $\begin{array}{c}\text { Overall } \\
55 \text { (RNA) } 89 \\
\quad \text { (NGS) }\end{array}$ & $\begin{array}{c}\text { gene expression } \\
\text { (PTEN-related encoding gene } \\
\text { TPTE) }\end{array}$ & $\begin{array}{l}\text { PTEN-related encoding gene } \\
\text { TPTE significantly } \\
\text { overexpressed in responders } \\
\text { but not in non-responders }\end{array}$ \\
\hline
\end{tabular}


Table 2. Phase II and phase III clinical trials with translational analyses exploring the predictive role of PTEN in HER2-positive breast cancer.

\begin{tabular}{|c|c|c|c|c|c|c|c|c|c|}
\hline $\begin{array}{l}\text { Treatment } \\
\text { Strategy }\end{array}$ & Agent & Trial & Phase & Population & Setting & Arms & $\begin{array}{c}\text { N pts } \\
\text { (Translational } \\
\text { Analysis) }\end{array}$ & PTEN Analysis & Main Results \\
\hline \multirow{6}{*}{$\begin{array}{c}\text { Single } \\
\text { anti-HER2 } \\
\text { blockade }\end{array}$} & \multirow{4}{*}{ Trastuzumab } & $\begin{array}{l}\text { NCT00133796 } \\
{[43]}\end{array}$ & II & EBC & Neoadjuvant & $\mathrm{CT}+$ Trastuzumab & 35 & $\begin{array}{l}\text { IHC (protein level: } 0 \text { versus } \\
1+\text { versus } 2+\text { versus } 3+\text { ) }\end{array}$ & $\begin{array}{c}\text { pCR rates in low-PTEN } \\
\text { versus high-PTEN: } \\
15.4 \% \text { versus } 44.4 \%\end{array}$ \\
\hline & & $\begin{array}{c}\text { GeparQuattro } \\
{[44]}\end{array}$ & III & EBC & Neoadjuvant & $\begin{array}{c}\text { CT (anthra-taxane) + } \\
\text { Trastuzumab } \\
2 \text { arms with CT } \\
\text { (anthra-taxane-cape) + } \\
\text { Trastuzumab }\end{array}$ & Overall 108 & $\begin{array}{l}\text { automated quantitative } \\
\text { immunofluorescence } \\
\text { (PTEN-low <60.1) }\end{array}$ & $\begin{array}{c}\mathrm{pCR} \text { rates in } \\
\text { high-PTEN versus } \\
\text { low-low PTEN: } 57.1 \% \\
\text { versus } 27.6 \%\end{array}$ \\
\hline & & N9831 [28] & III & EBC & Adjuvant & $\begin{array}{c}\mathrm{CT} \text { alone } \\
\mathrm{CT}+\text { sequential trastuzumab } \\
\mathrm{CT}+\text { concurrent } \\
\text { trastuzumab }\end{array}$ & $\begin{array}{l}601 \\
650 \\
551\end{array}$ & $\begin{array}{l}\text { IHC (PTEN+: }>0 \% \text { invasive } \\
\text { cells with } \geq 1+\text { cytoplasmatic } \\
\text { staining; examination of } \\
\text { alternate cut-points) }\end{array}$ & $\begin{array}{l}\text { No predictive role with } \\
\text { different cut-points } \\
\text { (DFS) }\end{array}$ \\
\hline & & $\begin{array}{c}\text { BCIRG-006 } \\
\text { [33] }\end{array}$ & III & EBC & Adjuvant & $\begin{array}{c}\text { CT (anthra) + Trastuzumab } \\
\text { CT (non-anthra) + } \\
\text { Trastuzumab } \\
\text { CT }\end{array}$ & $\begin{array}{l}402 \\
405 \\
394\end{array}$ & $\begin{array}{l}\text { IHC clone } 9559 \text { Cell } \\
\text { Signaling Technology } \\
\text { (protein level: } 0 \text { versus } 1+ \\
\text { versus } 2+\text { versus } 3+\text { ) }\end{array}$ & $\begin{array}{l}\text { No predictive role (DFS, } \\
\text { OS) }\end{array}$ \\
\hline & \multirow[t]{2}{*}{ Lapatinib } & $\begin{array}{c}\text { EGF103009 } \\
{[45]}\end{array}$ & II & $\mathrm{MBC}$ & $\begin{array}{l}\text { Refractory or } \\
\text { recurrent after } \\
\text { anthracycline- } \\
\text { containing } \\
\text { regimen in the } \\
\text { adjuvant or } \\
\text { metastatic setting }\end{array}$ & Lapatinib & 30 & $\begin{array}{c}\text { IHCclone by Cascade } \\
\text { Bioscience (protein level: } 0 \\
\text { versus } 1+\text { versus } 2+\text { versus } \\
3+\text { ) }\end{array}$ & $\begin{array}{l}\text { No predictive role } \\
\text { (ORR) }\end{array}$ \\
\hline & & $\begin{array}{c}\text { EGF104535 } \\
{[31]}\end{array}$ & III & MBC & First-line & $\begin{array}{l}\mathrm{CT}+\text { Lapatinib } \\
\mathrm{CT}+\text { placebo }\end{array}$ & $\begin{array}{l}180 \\
175\end{array}$ & $\begin{array}{l}\text { IHC clone 138G6 Cell } \\
\text { Signaling Technology } \\
\text { (protein level: } 0 \text { versus } 1+ \\
\text { versus } 2+\text { versus } 3+\text {; two } \\
\text { alternative cutoffs for } \\
\text { PTEN-low: } 0 / 1+\text { or } 0 \text { ) }\end{array}$ & $\begin{array}{l}\text { No predictive role (PFS, } \\
\text { OS) }\end{array}$ \\
\hline
\end{tabular}


Table 2. Cont.

\begin{tabular}{|c|c|c|c|c|c|c|c|c|c|}
\hline $\begin{array}{l}\text { Treatment } \\
\text { Strategy }\end{array}$ & Agent & Trial & Phase & Population & Setting & Arms & $\begin{array}{c}\mathbf{N} \text { pts } \\
\text { (Translational } \\
\text { Analysis) }\end{array}$ & PTEN Analysis & Main Results \\
\hline \multirow{6}{*}{$\begin{array}{c}\text { Dual } \\
\text { anti-HER2 } \\
\text { blockade }\end{array}$} & \multirow[t]{4}{*}{$\begin{array}{r}\text { Trastuzumab } \\
+ \text { Pertuzumab }\end{array}$} & Cleopatra [30] & III & MBC & First-line & $\begin{array}{c}\mathrm{CT}+\text { Trastuzumab }+ \\
\text { Pertuzumab } \\
\mathrm{CT}+\text { Trastuzumab + Placebo }\end{array}$ & $\begin{array}{l}\text { Overall } \\
497\end{array}$ & $\begin{array}{l}\text { IHC clone AF847; R and D } \\
\text { Systems (modified H-scores: } \\
0-400, \text { for membrane, } \\
\text { cytoplasm and nucleus; low } \\
\text { versus high: median value } \\
\text { adopted as cutoff) }\end{array}$ & $\begin{array}{l}\text { No predictive role (PFS, } \\
\text { OS) }\end{array}$ \\
\hline & & $\begin{array}{c}\text { TRYPHAENA } \\
{[46]}\end{array}$ & II & EBC & Neoadjuvant & $\begin{array}{c}3 \text { arms of Pertuzumab }+ \\
\text { Trastuzumab }+ \text { CT (different } \\
\text { CT regimens) }\end{array}$ & Overall 225 & $\begin{array}{l}\text { IHC clone by Cell Signalling } \\
\text { (modified H-score: } 0-400, \\
\text { nuclear compartment; low } \\
\text { versus high: median value } \\
\text { adopted as cutoff) }\end{array}$ & $\begin{array}{l}\text { No predictive role } \\
\quad(\mathrm{pCR})\end{array}$ \\
\hline & & $\begin{array}{l}\text { NeoSphere } \\
{[47]}\end{array}$ & II & EBC & Neoadjuvant & $\begin{array}{c}\text { CT + Trastuzumab } \\
\text { CT + Pertuzumab }+ \\
\text { Trastuzumab } \\
\text { CT + Pertuzumab } \\
\text { Trastuzumab + Pertuzumab }\end{array}$ & $\begin{array}{l}95 \\
95 \\
85 \\
98\end{array}$ & $\begin{array}{l}\text { IHC (modified H-score: } \\
0-400, \text { cytoplasmatic } \\
\text { compartment; low versus } \\
\text { high: median value adopted } \\
\text { as cutoff) }\end{array}$ & $\begin{array}{l}\text { No predictive role } \\
\quad(\mathrm{pCR})\end{array}$ \\
\hline & & Aphinity [34] & III & EBC & Adjuvant & $\begin{array}{c}\mathrm{CT}+\text { Trastuzumab }+ \\
\text { Pertuzumab } \\
\mathrm{CT}+\text { Trastuzumab + Placebo }\end{array}$ & $\begin{array}{l}\text { Overall } \\
\quad 939\end{array}$ & $\begin{array}{l}\text { DNA analysis for PI3K } \\
\text { pathway alterations }\end{array}$ & $\begin{array}{c}\text { Trend towards } \\
\text { decreased benefit from } \\
\text { pertuzumab in patients } \\
\text { with } \\
\text { PTEN/AKT/PIK3CA } \\
\text { alterations (NS) }\end{array}$ \\
\hline & \multirow{2}{*}{$\begin{array}{l}\text { Trastuzumab } \\
\text { + Lapatinib }\end{array}$} & $\begin{array}{c}\text { NCT00206427 } \\
\text { [43] }\end{array}$ & II & EBC & Neoadjuvant & $\underset{\text { Lapatinib }}{\mathrm{CT}+\text { Trastuzumab }^{\text {Tap }}}+$ & 49 & $\begin{array}{c}\text { IHC clone AF847, R and D } \\
\text { Systems (protein level: } 0 \\
\text { versus } 1+\text { versus } 2+\text { versus } \\
3+\text { ) }\end{array}$ & $\begin{array}{c}\text { pCR rates in low PTEN } \\
\text { versus normal-PTEN: } \\
92.3 \% \text { versus } 41.2 \%\end{array}$ \\
\hline & & $\begin{array}{c}\text { NCT00524303 } \\
{[48]}\end{array}$ & II & EBC & Neoadjuvant & $\begin{array}{c}\mathrm{CT}+\text { Trastuzumab } \\
\text { CT + Lapatinib } \\
\mathrm{CT}+\text { Trastuzumab }+ \\
\text { Lapatinib }\end{array}$ & $\begin{array}{c}\text { Overall } \\
49\end{array}$ & NA & $\begin{array}{l}\text { No predictive role } \\
\qquad(\mathrm{pCR})\end{array}$ \\
\hline
\end{tabular}


Table 2. Cont.

\begin{tabular}{|c|c|c|c|c|c|c|c|c|c|}
\hline $\begin{array}{l}\text { Treatment } \\
\text { Strategy }\end{array}$ & Agent & Trial & Phase & Population & Setting & Arms & $\begin{array}{c}\mathbf{N p t s} \\
\text { (Translational } \\
\text { Analysis) }\end{array}$ & PTEN Analysis & Main Results \\
\hline & & $\begin{array}{c}\text { Neo-ALTTO } \\
\text { [29] }\end{array}$ & III & $\mathrm{EBC}$ & Neoadjuvant & $\begin{array}{c}\text { Trastuzumab } \\
\text { Lapatinib } \\
\text { Trastuzumab + Lapatinib }\end{array}$ & Overall 429 & $\begin{array}{l}\text { IHC clone } 6 \mathrm{H} 2.1 \mathrm{DAKO} \text { and } \\
\text { clone } 138 \mathrm{G} 6 \text { Cell Signaling } \\
\text { Technology (H score system: } \\
\text { PTEN-low H-score }<50 \text { in } \\
\text { invasive tumor cell } \\
\text { compartment; analysis of } \\
\text { alternative cut-point H-score } \leq \\
10 ; 2 \text { observers) }\end{array}$ & No predictive role (pCR) \\
\hline & & $\begin{array}{c}\text { CHER-LOB } \\
{[49]}\end{array}$ & II & ЕBC & Neoadjuvant & $\begin{array}{c}\text { CT+ Trastuzumab } \\
\text { CT + Lapatinib } \\
\text { CT + Trastuzumab }+ \\
\text { Lapatinib }\end{array}$ & $\begin{array}{c}\text { Overall } \\
121\end{array}$ & $\begin{array}{c}\text { IHC clone } 28 \mathrm{H} 6 \text { Novocastra } \\
\text { (PTEN loss: staining }<10 \% \\
\text { cancer cells) }\end{array}$ & No predictive role (pCR) \\
\hline & & $\begin{array}{c}\text { ICORG 10-05 } \\
{[50]}\end{array}$ & II & ЕBC & Neoadjuvant & $\begin{array}{c}\text { CT + Trastuzumab } \\
\text { CT + Lapatinib } \\
\text { CT + Trastuzumab }+ \\
\text { Lapatinib }\end{array}$ & $\begin{array}{c}21 \\
6 \\
18\end{array}$ & $\begin{array}{c}\text { IHC clone } 6 \mathrm{H} 2.1 \text { Dako (protein } \\
\text { level: } 0 \text { versus } 1+\text { versus } 2+ \\
\text { versus } 3+\text { ) }\end{array}$ & No predictive role (pCR) \\
\hline & & $\begin{array}{c}\text { TBCRC006 } \\
\text { [51] }\end{array}$ & II & EBC & Neoadjuvant & Trastuzumab + Lapatinib & 59 & $\begin{array}{l}\text { IHC clone D4.3 Cell Signaling } \\
\text { (H-score. PTEN low versus high: } \\
\text { H-score }<100 \text { versus } \geq 100 \text { ) }\end{array}$ & $\begin{array}{c}\text { pCR rates in high-PTEN } \\
\text { versus low-PTEN: } 32 \% \\
\text { versus } 9 \%\end{array}$ \\
\hline \multirow[t]{3}{*}{$\begin{array}{c}\text { Drug } \\
\text { conjugate }\end{array}$} & \multirow[t]{3}{*}{ TDM1 } & Emilia [52] & III & $\mathrm{MBC}$ & $\begin{array}{l}\text { Previous treatment } \\
\text { with Trastuzumab } \\
\text { and a taxane }\end{array}$ & $\begin{array}{c}\text { TDM1 } \\
\text { CT + Lapatinib }\end{array}$ & $\begin{array}{l}134 \\
137\end{array}$ & $\begin{array}{l}\text { IHC clone 138G6 Cell Signaling } \\
\text { Technology (cytoplasmic PTEN } \\
\text { expression: none versus } \\
\text { decreased versus slightly } \\
\text { decreased versus equivalent } \\
\text { versus increased) }\end{array}$ & $\begin{array}{l}\text { Absent or decreased } \\
\text { tumor PTEN expression } \\
\text { associated PFS benefit } \\
\text { with T-DM1 relative to } \\
\text { that with CT + Lapatinib }\end{array}$ \\
\hline & & TH3RESA [32] & III & $\mathrm{MBC}$ & $\begin{array}{l}\geq 2 \text { previous } \\
\text { anti-HER2 regimens } \\
\text { (including } \\
\text { Trastuzumab and } \\
\text { Lapatinib in the } \\
\text { advanced setting) } \\
\end{array}$ & $\begin{array}{l}\text { TDM1 } \\
\text { TPC }\end{array}$ & $\begin{array}{l}247 \\
111\end{array}$ & $\begin{array}{c}\text { IHC clone 138G6 Cell Signaling } \\
\text { Technology (H-score: } 0-400 ; \text { low } \\
\text { versus high: median adopted as } \\
\text { cutoff }=200)\end{array}$ & No predictive role (PFS) \\
\hline & & Marianne [53] & III & $\mathrm{MBC}$ & First-line & $\begin{array}{c}\text { CT }+ \text { Trastuzumab } \\
\text { TDM1 } \\
\text { TDM1 + Pertuzumab }\end{array}$ & $\begin{array}{l}182 \\
181 \\
179\end{array}$ & $\begin{array}{l}\text { IHC clone 138G6 Cell Signaling } \\
\text { Technology (protein level: } 0 \\
\text { versus 1+ versus 2+ versus } 3+; \\
\text { H-score: median value adopted } \\
\text { as cutoff, cytoplasmatic } \\
\text { compartment) }\end{array}$ & No predictive role (PFS) \\
\hline
\end{tabular}

Abbreviations: EBC, early breast cancer; CT, chemotherapy; IHC, immunohistochemistry; pCR, pathologic complete response; DFS, disease-free survival; anthra, anthracycline-containing regimen; non-anthra, non-anthracycline containing regimen; OS; overall survival; cape, Capecitabine; MBC, metastatic breast cancer; PFS, progression-free survival, ORR, overall response rate; NA, not applicable; NS, not-significant; TDM1, trastuzumab-emtansine; TPC, treatment of physician's choice. 


\subsection{1. mTOR Inhibitors}

On the basis of the preclinical observation that PTEN loss may increase tumor sensitivity to mTOR inhibition $[14,54]$, the predictive potential of PTEN in BC patients has been explored in the context of several clinical trials testing the mTOR inhibitor Everolimus for metastatic BC, with conflicting results. In detail, in the TAMRAD non-comparative phase II trial, $\mathrm{HR}+$ metastatic $\mathrm{BC}$ patients were randomized to receive either Tamoxifen plus Everolimus or Tamoxifen alone. Biomarker analysis failed to report any predictive association between PTEN loss (assessed both by immunohistochemistry and next-generation sequencing analysis) and clinical benefit from the addition of Everolimus to Tamoxifen [35]. Similarly, in the BOLERO-2 phase III trial, randomizing HR+/HER2- metastatic BC patients to receive Everolimus plus Exemestane versus placebo plus Exemestane, PTEN mutations or low PTEN protein expression assessed on tumor samples were not associated with Everolimus benefit [36]. Conversely, the pooled analysis of the BOLERO-1 and BOLERO-3 phase III trials (testing the addition of Everolimus to Trastuzumab plus Paclitaxel and Trastuzumab plus Vinorelbine, respectively) revealed a potential predictive role of PTEN status with regard to Everolimus benefit. In particular, Andrè et al., reported that patients harboring low/absent PTEN tumor expression by immunohistochemistry or a hyperactive PI3K pathway (defined as either low/absent PTEN expression or PTEN mutations or PIK3CA activating mutations or AKT1 E17K mutations) experienced a significant progression-free survival (PFS) improvement with the addition of Everolimus to chemotherapy plus Trastuzumab, as compared to patients without these alterations [55].

\subsubsection{AKT Inhibitors}

Interestingly, phase II trials evaluating the addition of AKT inhibitors to first-line Paclitaxel for triple-negative BC consistently reported an association between PI3K pathway deregulation (including PTEN alterations) and response to AKT inhibitors treatment both in advanced and early setting.

In particular, in the LOTUS trial, the addition of Ipatasertib to Paclitaxel significantly prolonged PFS of metastatic triple-negative BC patients (HR 0-60, 95\% CI 0.37-0.98, $p=0.037$ ). Patients were classified according to: PTEN expression by immunohistochemistry (PTEN-low or PTEN-high) and PIK3CA/AKT1/PTEN genomic alterations (based on the presence of either PIK3CA, AKT1 or PTEN mutations). In the subgroup of PTEN-low patients, there was no statistically significant difference in PFS between the two arms. However, in the PIK3CA/Akt1/PTEN-altered subgroup, the benefit of adding Ipatasertib to Paclitaxel was significant (HR 0-44, 95\% CI 0.20-0.99). These data may suggest that the definition of PTEN functional status through mutations and copy number losses by next-generation sequencing may provide more biologically and clinically relevant information than the evaluation of PTEN protein expression by immunohistochemistry and that a comprehensive assessment of the PI3K pathway's functional status may be of relevance rather than focusing on single alterations [37]. Similarly, in the PAKT trial, Schmid et al. reported that the PFS prolongation observed with Capivasertib plus Paclitaxel versus Paclitaxel plus placebo in triple-negative metastatic BC was mainly driven by the PIK3CA/Akt1/PTEN-altered population, since no beneficial effect from the addition of Capivasertib to Paclitaxel was observed in patients with PIK3CA/Akt1/PTEN non-altered tumors [39].

A similar predictive role of PTEN status has been suggested also in triple-negative early BC in the context of the FARLAINE trial, where the combination of Ipatasertib plus Paclitaxel has been compared to Paclitaxel plus placebo in the neoadjuvant setting. Oliveira et al., reported higher pathological complete response (pCR) rates with Ipatasertib both in PTEN-low (assessed by immunohistochemistry) and PIK3CA/Akt1/PTEN-altered (by next-generation sequencing) subgroups, as compared to patients without these alterations. In addition, higher complete response rates by magnetic resonance imaging were observed in the same biomarker-selected subgroups of patients [38].

The predictive role of PTEN status with respect to response to AKT inhibition has also been explored in $\mathrm{HR}+$ metastatic BC. In particular, recently presented results from the phase $\mathrm{Ib}-\mathrm{II}$ Faktion trial suggested that the activation of the PI3K/Akt/PTEN pathway (defined as activating PIK3CA 
mutations in tumor tissue/blood or PTEN null by immunohistochemistry in primary or metastatic tumor tissue) did not affect sensitivity to Capivasertib, since a beneficial effect from the addition of the Akt inhibitor to Fulvestrant in HR+ metastatic BC was observed in both PI3K/Akt/PTEN activated and non-activated tumors [40].

\subsubsection{Anti-HER2-Based Treatments}

In HER2 + BC, low or absent PTEN expression has been reported in 20-25\% of cases [56]. Although HER2 status remains the only recognized and validated predictive biomarker to select patients for anti-HER2-based treatment, a growing body of evidence suggests that biomarkers involved in the PI3K pathway may have a role in affecting the response to HER2-targeted therapies.

\subsection{Single Anti-HER2 Blockade}

\subsubsection{Trastuzumab}

\section{Early BC: Neoadjuvant Setting}

Based on preclinical and clinical data suggesting that PTEN functional status may contribute to resistance to Trastuzumab-based therapy $[10,57,58]$, such a potential predictive role of PTEN has been explored in the context of several clinical trials in HER2+ early BC, with non-univocal results.

In detail, Dave et al. reported significantly lower pCR rates after neoadjuvant Trastuzumab plus chemotherapy in HER2+ BC patients with low PTEN expression by immunohistochemistry, as compared to those with higher PTEN protein levels [43].

Consistently, it has recently been reported that in the HER2+ population of the GEPARQuattro phase III trial, low PTEN expression assessed by immunofluorescence was significantly and independently associated with lower $\mathrm{pCR}$ rates after neoadjuvant therapy with chemotherapy plus Trastuzumab in the overall HER2+ population and HR+/HER2+ subgroup [44].

\section{Early BC: Adjuvant Setting}

Two randomized phase III trials of chemotherapy +/- Trastuzumab for early BC failed to report an association between PTEN status and adjuvant Trastuzumab benefit in terms of either DFS [28,33] or OS [33].

\subsubsection{Lapatinib}

\section{Metastatic BC}

The potential role of PTEN status in predicting response or resistance to Lapatinib-based therapy has been investigated in advanced BC in the context of several prospective cohorts of HER2+ BC patients, with conflicting results.

In a prospective global Capecitabine plus Lapatinib Expanded Access Program for patients progressing on Trastuzumab plus chemotherapy-based regimens, loss of PTEN by immunohistochemistry was associated with lower clinical benefit rates to Capecitabine plus Lapatinib than those with PTEN-positive tumors [59]. However, this observation was not confirmed in randomized phase II or III trials, where PTEN deficiency assessed by immunohistochemistry was not found to preclude a response to Lapatinib plus endocrine therapy or Lapatinib plus Paclitaxel in metastatic BC $[31,45]$. 


\subsection{Dual Anti-HER2 Blockade}

\subsubsection{Trastuzumab and Pertuzumab}

Early BC: Neoadjuvant Setting

The potential role of PTEN status in predicting the benefit from HER2-dual blockade with Trastuzumab and Pertuzumab has been investigated in the context of neoadjuvant clinical trials. However, they did not report any correlation between PTEN protein expression (assessed by immunohistochemistry) and pCR rates after Pertuzumab plus Trastuzumab-based therapy $[46,47]$.

\section{Early BC: Adjuvant Setting}

The recently presented translational analysis of the Aphinity trial (adjuvant treatment with either chemotherapy plus Trastuzumab and Pertuzumab or chemotherapy plus Trastuzumab and placebo) revealed a trend towards decreased benefit from the addition of Pertuzumab to adjuvant Trastuzumab plus chemotherapy in patients whose tumors harbored PI3K/Akt/PTEN alterations, such as PTEN homozygous deletion or any PTEN alteration by DNA analysis, thus further highlighting that a more comprehensive evaluation of PTEN status by genomic analysis may help with better capturing the biological relevance of PTEN in affecting response to anti-HER2-based therapy than the mere protein expression by immunohistochemistry [34].

\section{Metastatic BC}

The translational analysis of the Cleopatra study revealed that the survival benefit (PFS and OS) observed with the addition of Pertuzumab to Trastuzumab plus chemotherapy as first-line treatment for metastatic BC patients was independent from PTEN status [30].

\subsubsection{Trastuzumab and Lapatinib}

\section{Early BC: Neoadjuvant Setting}

Although a positive association between low PTEN expression and $\mathrm{pCR}$ rates after neoadjuvant therapy with Lapatinib plus Trastuzumab and chemotherapy was initially suggested by Dave et al. [39], available data from phase II and III neoadjuvant clinical trials testing chemotherapy plus single or dual anti-HER2 blockade with Trastuzumab and Lapatinib failed to demonstrate significant differences in pCR rates according to PTEN status overall and per treatment arm [29,48-50].

In the context of the TBCRC 006 trial, low PTEN expression has been counterintuitively associated with lower $\mathrm{PCR}$ rates after dual HER2 blockade with Lapatinib and Trastuzumab without chemotherapy, as compared to higher PTEN expression [51].

Although these data highlight that solid evidence is missing on the actual role of PTEN in affecting the response to HER2 dual blockade, available evidence suggest that the PI3K/Akt pathway may actually retain a role in affecting the response to anti-HER2-based therapy. Indeed, a recent pooled analysis of almost 1000 HER2 + early BC patients from five randomized clinical trials of neoadjuvant treatment with chemotherapy in addition to either Trastuzumab, Lapatinib or the combination of them, reported lower pCR rates in PIK3CA mutant group as compared to the wild-type group, especially in the HR+ subpopulation and in patients receiving the dual HER2 blockade [60]. These data emphasize that a further deepening of the potential predictive value of the PI3K/Akt pathway with regard to anti-HER2-based therapy might be of great interest.

\subsubsection{Trastuzumab-Emtansine (TDM1)}

Biomarker analyses of randomized trials evaluating TDM1 for the treatment of HER2+ metastatic BC consistently report that PTEN was not predictive for TDM1 benefit [32,53]. However, a trend towards a more pronounced PFS benefit in patients with decreased or absent PTEN protein expression 
and treated with TDM1 relative to Capecitabine-Lapatinib has been reported, as compared to patients with higher PTEN expression levels [52].

Taken together, the available data, although preliminary, seem to generate the hypothesis that PTEN loss may potentially preclude response to Trastuzumab- or Lapatinib-based therapy, but not to TDM1.

\subsubsection{Immunotherapy}

As suggested by preclinical models, PTEN loss may induce PD-L1 overexpression, thus hinting at a potential role of PTEN in affecting the response to immune-checkpoint inhibition.

Interestingly, Sousa et al. investigated PTEN genomic status in triple-negative metastatic BC patients treated with PD1/PDL1 immune checkpoint inhibitors-mostly associated with chemotherapy-in the context of several clinical trials (NCT02447003, NCT01375842, NCT02513472, NCT01633970) at Dana Farber Institute, reporting that PTEN alterations (mutations and 1 or 2 copy number loss) were significantly and independently associated with lower overall-response rate and worse PFS and OS rates with respect to patients without these molecular alterations [61].

\subsubsection{Endocrine Therapy}

In a randomized non comparative phase II trial of Anastrozole or Fulvestrant as neoadjuvant endocrine therapy for post-menopausal $\mathrm{HR}+\mathrm{BC}$ patients, responders were found to harbor a significant overexpression of PTEN-related encoding gene (TPTE), as compared to non-responders, and this upregulation was maintained even after months of neoadjuvant endocrine therapy. Of course, this observation warrants further investigation [42].

\subsubsection{PARP-Inhibitor}

Emerging evidence suggests that PTEN is involved in other functions beyond the PI3K/Akt pathway, namely DNA damage repair via homologous recombination $[13,62,63]$.

Interestingly, in a phase II trial testing the PARP-inhibitor Talazoparib as monotherapy for BRCA(Breast Related Cancer Antigen) 1/2 wild-type HER2- BC patients, authors reported that tumors which exhibited response or stability harbored gene mutations in the homologous recombination pathway, including PTEN gene mutations [41].

\section{Conclusions}

Although there is some evidence for an association between PTEN functional status and both clinical outcome and response to various treatments, robust evidence is missing to properly establish its actual prognostic and/or predictive role in BC.

One of the major limitations is that PTEN assessment lacks consistency and reproducibility across studies in terms of type of assay (immunohistochemistry versus next-generation sequencing), antibodies for immunohistochemistry testing, scoring system (e.g., H-score, protein levels, \% of positive cells), cutoffs for PTEN-loss definition, and origin of tumor samples (primary versus metastatic samples).

In addition, the clinical utility of PTEN-loss has been explored in cohorts of patients treated with considerably heterogeneous regimens, thus further increasing the complexity of the interpretation of available results.

To conclude, although currently unfulfilled, the potential of PTEN as a biomarker in BC is promising and deserves further investigation. Indeed, the optimal inter-observer and inter-antibody agreement of PTEN assessment, reported in the context of a prospective cohort of BC patients enrolled in a phase III randomized trial [29], appears reassuring and encourages further efforts in the context of well-designed and well-conducted biomarker-driven trials. Moreover, the recent finding that PTEN seems to be implicated in the control of tumor microenvironment and immune system leads to future potential perspectives for an immunotherapy-based therapeutic strategy. 
Funding: This research received no external funding.

Conflicts of Interest: Maria Vittoria Dieci: Eli Lilly and Company, Genomic Health (C/A), Eli Lilly and Company, Celgene (SAB). The other authors declare no conflict of interest.

\section{References}

1. Li, D.M.; Sun, H. TEP1, Encoded by a Candidate Tumor Suppressor Locus, is a Novel Protein Tyrosine Phosphatase Regulated by Transforming Growth Factor Beta. Cancer Res. 1997, 57, 2124-2129. [PubMed]

2. Steck, P.A.; Pershouse, M.A.; Jasser, S.A.; Yung, W.K.; Lin, H.; Ligon, A.H.; Langford, L.A.; Baumgard, M.L.; Hattier, T.; Davis, T.; et al. Identification of a Candidate Tumour Suppressor Gene, MMAC1, at Chromosome 10q23.3 that is Mutated in Multiple Advanced Cancers. Nat. Genet. 1997, 15, 356-362. [CrossRef] [PubMed]

3. Li, J.; Yen, C.; Liaw, D.; Podsypanina, K.; Bose, S.; Wang, S.I.; Puc, J.; Miliaresis, C.; Rodgers, L.; McCombie, R.; et al. PTEN, a Putative Protein Tyrosine Phosphatase Gene Mutated in Human Brain, Breast, and Prostate Cancer. Science 1997, 275, 1943-1947. [CrossRef] [PubMed]

4. Engelman, J.A.; Luo, J.; Cantley, L.C. The Evolution of Phosphatidylinositol 3-Kinases as Regulators of Growth and Metabolism. Nat. Rev. Genet. 2006, 7, 606-619. [CrossRef] [PubMed]

5. Shaw, R.J.; Cantley, L.C. Ras, PI(3)K and mTOR Signalling Controls Tumour Cell Growth. Nature 2006, 441, 424-430. [CrossRef] [PubMed]

6. Song, M.S.; Carracedo, A.; Salmena, L.; Song, S.J.; Egia, A.; Malumbres, M.; Pandolfi, P.P. Nuclear PTEN Regulates the APC-CDH1 Tumor-Suppressive Complex in a Phosphatase-Independent Manner. Cell 2011, 144, 187-199. [CrossRef] [PubMed]

7. Freeman, D.J.; Li, A.G.; Wei, G.; Li, H.H.; Kertesz, N.; Lesche, R.; Whale, A.D.; Martinez-Diaz, H.; Rozengurt, N.; Cardiff, R.D.; et al. PTEN Tumor Suppressor Regulates p53 Protein Levels and Activity through Phosphatase-Dependent and -Independent Mechanisms. Cancer Cell 2003, 3, 117-130. [CrossRef]

8. Ma, J.; Benitez, J.A.; Li, J.; Miki, S.; Ponte de Albuquerque, C.; Galatro, T.; Orellana, L.; Zanca, C.; Reed, R.; Boyer, A.; et al. Inhibition of Nuclear PTEN Tyrosine Phosphorylation Enhances Glioma Radiation Sensitivity through Attenuated DNA Repair. Cancer Cell 2019, 35, 816. [CrossRef]

9. Leslie, N.R.; Spinelli, L.; Tibarewal, P.; Zilidis, G.; Weerasinghe, N.; Lim, J.C.; Maccario, H.; Downes, C.P. Indirect Mechanisms of Carcinogenesis Via Downregulation of PTEN Function. Adv. Enzyme Regul. 2010, 50, 112-118. [CrossRef]

10. Song, M.S.; Salmena, L.; Pandolfi, P.P. The Functions and Regulation of the PTEN Tumour Suppressor. Nat. Rev. Mol. Cell Biol. 2012, 13, 283-296. [CrossRef]

11. Yehia, L.; Ngeow, J.; Eng, C. PTEN-Opathies: From Biological Insights to Evidence-Based Precision Medicine. J. Clin. Investig. 2019, 129, 452-464. [CrossRef] [PubMed]

12. Coughlin, C.M.; Johnston, D.S.; Strahs, A.; Burczynski, M.E.; Bacus, S.; Hill, J.; Feingold, J.M.; Zacharchuk, C.; Berkenblit, A. Approaches and Limitations of Phosphatidylinositol-3-Kinase Pathway Activation Status as a Predictive Biomarker in the Clinical Development of Targeted Therapy. Breast Cancer Res. Treat. 2010, 124, 1-11. [CrossRef] [PubMed]

13. Bazzichetto, C.; Conciatori, F.; Pallocca, M.; Falcone, I.; Fanciulli, M.; Cognetti, F.; Milella, M.; Ciuffreda, L. PTEN as a Prognostic/Predictive Biomarker in Cancer: An Unfulfilled Promise? Cancers 2019, 11, 435. [CrossRef] [PubMed]

14. Nagata, Y.; Lan, K.H.; Zhou, X.; Tan, M.; Esteva, F.J.; Sahin, A.A.; Klos, K.S.; Li, P.; Monia, B.P.; Nguyen, N.T.; et al. PTEN Activation Contributes to Tumor Inhibition by Trastuzumab, and Loss of PTEN Predicts Trastuzumab Resistance in Patients. Cancer Cell 2004, 6, 117-127. [CrossRef] [PubMed]

15. Stemke-Hale, K.; Gonzalez-Angulo, A.M.; Lluch, A.; Neve, R.M.; Kuo, W.L.; Davies, M.; Carey, M.; Hu, Z.; Guan, Y.; Sahin, A.; et al. An Integrative Genomic and Proteomic Analysis of PIK3CA, PTEN, and AKT Mutations in Breast Cancer. Cancer Res. 2008, 68, 6084-6091. [CrossRef] [PubMed]

16. Perez-Tenorio, G.; Alkhori, L.; Olsson, B.; Waltersson, M.A.; Nordenskjold, B.; Rutqvist, L.E.; Skoog, L.; Stal, O. PIK3CA Mutations and PTEN Loss Correlate with Similar Prognostic Factors and are Not Mutually Exclusive in Breast Cancer. Clin. Cancer Res. 2007, 13, 3577-3584. [CrossRef] [PubMed]

17. Alimonti, A.; Carracedo, A.; Clohessy, J.G.; Trotman, L.C.; Nardella, C.; Egia, A.; Salmena, L.; Sampieri, K.; Haveman, W.J.; Brogi, E.; et al. Subtle Variations in Pten Dose Determine Cancer Susceptibility. Nat. Genet. 2010, 42, 454-458. [CrossRef] [PubMed] 
18. Zhou, X.J.; Wu, J.; Shi, L.; Li, X.X.; Zhu, L.; Sun, X.; Qian, J.Y.; Wang, Y.; Wei, J.F.; Ding, Q. PTEN Expression is Upregulated by a RNA-Binding Protein RBM38 Via Enhancing its mRNA Stability in Breast Cancer. J. Exp. Clin. Cancer Res. 2017, 36, 149. [CrossRef]

19. Trimboli, A.J.; Cantemir-Stone, C.Z.; Li, F.; Wallace, J.A.; Merchant, A.; Creasap, N.; Thompson, J.C.; Caserta, E.; Wang, H.; Chong, J.L.; et al. Pten in Stromal Fibroblasts Suppresses Mammary Epithelial Tumours. Nature 2009, 461, 1084-1091. [CrossRef]

20. Majumder, S.; Jacob, S.T. Emerging Role of microRNAs in Drug-Resistant Breast Cancer. Gene Expr. 2011, 15, 141-151. [CrossRef]

21. Shen, H.; Wang, D.; Li, L.; Yang, S.; Chen, X.; Zhou, S.; Zhong, S.; Zhao, J.; Tang, J. MiR-222 Promotes Drug-Resistance of Breast Cancer Cells to Adriamycin Via Modulation of PTEN/Akt/FOXO1 Pathway. Gene 2017, 596, 110-118. [CrossRef] [PubMed]

22. Zhang, S.; Huang, W.C.; Li, P.; Guo, H.; Poh, S.B.; Brady, S.W.; Xiong, Y.; Tseng, L.M.; Li, S.H.; Ding, Z.; et al. Combating Trastuzumab Resistance by Targeting SRC, a Common Node Downstream of Multiple Resistance Pathways. Nat. Med. 2011, 17, 461-469. [CrossRef] [PubMed]

23. Tanaka, H.; Yoshida, M.; Tanimura, H.; Fujii, T.; Sakata, K.; Tachibana, Y.; Ohwada, J.; Ebiike, H.; Kuramoto, S.; Morita, K.; et al. The Selective Class I PI3K Inhibitor CH5132799 Targets Human Cancers Harboring Oncogenic PIK3CA Mutations. Clin. Cancer Res. 2011, 17, 3272-3281. [CrossRef] [PubMed]

24. Brachmann, S.M.; Hofmann, I.; Schnell, C.; Fritsch, C.; Wee, S.; Lane, H.; Wang, S.; Garcia-Echeverria, C.; Maira, S.M. Specific Apoptosis Induction by the Dual PI3K/mTor Inhibitor NVP-BEZ235 in HER2 Amplified and PIK3CA Mutant Breast Cancer Cells. Proc. Natl. Acad. Sci. USA 2009, 106, 22299-22304. [CrossRef] [PubMed]

25. Juric, D.; Castel, P.; Griffith, M.; Griffith, O.L.; Won, H.H.; Ellis, H.; Ebbesen, S.H.; Ainscough, B.J.; Ramu, A.; Iyer, G.; et al. Convergent Loss of PTEN Leads to Clinical Resistance to a PI(3)Kalpha Inhibitor. Nature 2015, 518, 240-244. [CrossRef] [PubMed]

26. Mittendorf, E.A.; Philips, A.V.; Meric-Bernstam, F.; Qiao, N.; Wu, Y.; Harrington, S.; Su, X.; Wang, Y.; Gonzalez-Angulo, A.M.; Akcakanat, A.; et al. PD-L1 Expression in Triple-Negative Breast Cancer. Cancer Immunol. Res. 2014, 2, 361-370. [CrossRef]

27. Luen, S.J.; Asher, R.; Lee, C.K.; Savas, P.; Kammler, R.; Dell'Orto, P.; Biasi, O.M.; Demanse, D.; JeBailey, L.; Dolan, S.; et al. Association of Somatic Driver Alterations with Prognosis in Postmenopausal, Hormone Receptor-Positive, HER2-Negative Early Breast Cancer: A Secondary Analysis of the BIG 1-98 Randomized Clinical Trial. JAMA Oncol. 2018, 4, 1335-1343. [CrossRef] [PubMed]

28. Perez, E.A.; Dueck, A.C.; McCullough, A.E.; Chen, B.; Geiger, X.J.; Jenkins, R.B.; Lingle, W.L.; Davidson, N.E.; Martino, S.; Kaufman, P.A.; et al. Impact of PTEN Protein Expression on Benefit from Adjuvant Trastuzumab in Early-Stage Human Epidermal Growth Factor Receptor 2-Positive Breast Cancer in the North Central Cancer Treatment Group N9831 Trial. J. Clin. Oncol. 2013, 31, 2115-2122. [CrossRef]

29. Nuciforo, P.G.; Aura, C.; Holmes, E.; Prudkin, L.; Jimenez, J.; Martinez, P.; Ameels, H.; de la Pena, L.; Ellis, C.; Eidtmann, H.; et al. Benefit to Neoadjuvant Anti-Human Epidermal Growth Factor Receptor 2 (HER2)-Targeted Therapies in HER2-Positive Primary Breast Cancer is Independent of Phosphatase and Tensin Homolog Deleted from Chromosome 10 (PTEN) Status. Ann. Oncol. 2015, 26, 1494-1500. [CrossRef]

30. Baselga, J.; Cortes, J.; Im, S.A.; Clark, E.; Ross, G.; Kiermaier, A.; Swain, S.M. Biomarker Analyses in CLEOPATRA: A Phase III, Placebo-Controlled Study of Pertuzumab in Human Epidermal Growth Factor Receptor 2-Positive, First-Line Metastatic Breast Cancer. J. Clin. Oncol. 2014, 32, 3753-3761. [CrossRef]

31. Xu, B.; Guan, Z.; Shen, Z.; Tong, Z.; Jiang, Z.; Yang, J.; DeSilvio, M.; Russo, M.; Leigh, M.; Ellis, C. Association of Phosphatase and Tensin Homolog Low and Phosphatidylinositol 3-Kinase Catalytic Subunit Alpha Gene Mutations on Outcome in Human Epidermal Growth Factor Receptor 2-Positive Metastatic Breast Cancer Patients Treated with First-Line Lapatinib Plus Paclitaxel Or Paclitaxel Alone. Breast Cancer Res. 2014, 16, 405. [PubMed]

32. Kim, S.B.; Wildiers, H.; Krop, I.E.; Smitt, M.; Yu, R.; Lysbet de Haas, S.; Gonzalez-Martin, A. Relationship between Tumor Biomarkers and Efficacy in TH3RESA, a Phase III Study of Trastuzumab Emtansine (T-DM1) Vs. Treatment of Physician's Choice in Previously Treated HER2-Positive Advanced Breast Cancer. Int. J. Cancer 2016, 139, 2336-2342. [CrossRef] [PubMed] 
33. Stern, H.M.; Gardner, H.; Burzykowski, T.; Elatre, W.; O’Brien, C.; Lackner, M.R.; Pestano, G.A.; Santiago, A.; Villalobos, I.; Eiermann, W.; et al. PTEN Loss is Associated with Worse Outcome in HER2-Amplified Breast Cancer Patients but is Not Associated with Trastuzumab Resistance. Clin. Cancer Res. 2015, 21, 2065-2074. [CrossRef] [PubMed]

34. Krop, I.; Paulson, J.; Campbell, C.; Kiermaier, A.; André, F.; Fumagalli, D.; de Haas, S.; Salgado, R.; Loibla, S.; Bailey, A.; et al. Genomic Correlates of Response to Adjuvant Trastuzumab and Pertuzumab in HER2+ Breast Cancer: Biomarker Analysis of the APHINITY Trial. In Proceedings of the ASCO 2017, Chicago, IL, USA, 31 May-4 June 2017.

35. Treilleux, I.; Arnedos, M.; Cropet, C.; Wang, Q.; Ferrero, J.M.; Abadie-Lacourtoisie, S.; Levy, C.; Legouffe, E.; Lortholary, A.; Pujade-Lauraine, E.; et al. Translational Studies within the TAMRAD Randomized GINECO Trial: Evidence for mTORC1 Activation Marker as a Predictive Factor for Everolimus Efficacy in Advanced Breast Cancer. Ann. Oncol. 2015, 26, 120-125. [CrossRef] [PubMed]

36. Hortobagyi, G.N.; Chen, D.; Piccart, M.; Rugo, H.S.; Burris, H.A., 3rd; Pritchard, K.I.; Campone, M.; Noguchi, S.; Perez, A.T.; Deleu, I.; et al. Correlative Analysis of Genetic Alterations and Everolimus Benefit in Hormone Receptor-Positive, Human Epidermal Growth Factor Receptor 2-Negative Advanced Breast Cancer: Results from BOLERO-2. J. Clin. Oncol. 2016, 34, 419-426. [CrossRef] [PubMed]

37. Kim, S.B.; Dent, R.; Im, S.A.; Espie, M.; Blau, S.; Tan, A.R.; Isakoff, S.J.; Oliveira, M.; Saura, C.; Wongchenko, M.J.; et al. Ipatasertib Plus Paclitaxel Versus Placebo Plus Paclitaxel as First-Line Therapy for Metastatic Triple-Negative Breast Cancer (LOTUS): A Multicentre, Randomised, Double-Blind, Placebo-Controlled, Phase 2 Trial. Lancet Oncol. 2017, 18, 1360-1372. [CrossRef]

38. Oliveira, M.; Saura, C.; Nuciforo, P.; Calvo, I.; Andersen, J.; Passos-Coelho, J.L.; Gil Gil, M.; Bermejo, B.; Patt, D.A.; Ciruelos, E.; et al. FAIRLANE, a Double-Blind Placebo-Controlled Randomized Phase II Trial of Neoadjuvant Ipatasertib Plus Paclitaxel for Early Triple-Negative Breast Cancer. Ann. Oncol. 2019, 30, 1289-1297. [CrossRef] [PubMed]

39. Schmid, P.; Abraham, J.; Chan, S.; Wheatley, D.; Brunt, M. AZD5363 Plus Paclitaxel Versus Placebo Plus Paclitaxel as First-Line Therapy for Metastatic Triple-Negative Breast Cancer (PAKT): A Randomized, Double-Blind, Placebo-Controlled, Phase II Trial. J. Clin. Oncol. 2018, 36, 1007. [CrossRef]

40. Jones, R.; Carucci, M.; Casbard, A.; Butler, A.; Alchami, F.; Bale, C.; Bezecny, P.; Joffe, J.; Moon, S.; Twelves, C.; et al. Capivasertib (AZD5363) Plus Fulvestrant Versus Placebo Plus Fulvestrant After Relapse Or Pregression on an Aromatase Inhibitor in Metastatic ER Positive Breast Cancer (FAKTION): A Ramdomized Double-Blind Placebo-Controlled Phase II Trial. In Proceedings of the ASCO 2019, Chicago, IL, USA, 31 May-4 June 2019.

41. Gruber, J.; Afghahi, A.; Hatton, A.; Scott, D.; McMilan, A.; Ford, J.; Telli, M. Talazoparib Beyond BRCA: A Phase II Trial of Talazoparib Monotherapy in BRCA 1 and BRCA2 Wild-Type Patients with Advanced HER2negative Breast Cancer or Other Solid Tumors with a Mutation in Homologous Recombination Pathway Genes. In Proceedings of the ASCO 2019, Chicago, IL, USA, 31 May-4 June 2019.

42. Liang, X.; Briaux, A.; Becette, V.; Benoist, C.; Boulai, A.; Chemlali, W.; Schnitzler, A.; Baulande, S.; Rivera, S.; Mouret-Reynier, M.A.; et al. Molecular Profiling of Hormone Receptor-Positive, HER2-Negative Breast Cancers from Patients Treated with Neoadjuvant Endocrine Therapy in the CARMINA 02 Trial (UCBG-0609). J. Hematol. Oncol. 2018, 11, 124. [CrossRef]

43. Dave, B.; Migliaccio, I.; Gutierrez, M.C.; Wu, M.F.; Chamness, G.C.; Wong, H.; Narasanna, A.; Chakrabarty, A.; Hilsenbeck, S.G.; Huang, J.; et al. Loss of Phosphatase and Tensin Homolog Or Phosphoinositol-3 Kinase Activation and Response to Trastuzumab Or Lapatinib in Human Epidermal Growth Factor Receptor 2-Overexpressing Locally Advanced Breast Cancers. J. Clin. Oncol. 2011, 29, 166-173. [CrossRef]

44. Loibl, S.; Darb-Esfahani, S.; Huober, J.; Klimowicz, A.; Furlanetto, J.; Lederer, B.; Hartmann, A.; Eidtmann, H.; Pfitzner, B.; Fasching, P.A.; et al. Integrated Analysis of PTEN and p4EBP1 Protein Expression as Predictors for pCR in HER2-Positive Breast Cancer. Clin. Cancer Res. 2016, 22, 2675-2683. [CrossRef] [PubMed]

45. Johnston, S.; Trudeau, M.; Kaufman, B.; Boussen, H.; Blackwell, K.; LoRusso, P.; Lombardi, D.P.; Ben Ahmed, S.; Citrin, D.L.; DeSilvio, M.L.; et al. Phase II Study of Predictive Biomarker Profiles for Response Targeting Human Epidermal Growth Factor Receptor 2 (HER-2) in Advanced Inflammatory Breast Cancer with Lapatinib Monotherapy. J. Clin. Oncol. 2008, 26, 1066-1072. [CrossRef] [PubMed] 
46. Schneeweiss, A.; Chia, S.; Hegg, R.; Tausch, C.; Deb, R.; Ratnayake, J.; McNally, V.; Ross, G.; Kiermaier, A.; Cortes, J. Evaluating the Predictive Value of Biomarkers for Efficacy Outcomes in Response to Pertuzumaband Trastuzumab-Based Therapy: An Exploratory Analysis of the TRYPHAENA Study. Breast Cancer Res. 2014, 16, R73. [CrossRef] [PubMed]

47. Bianchini, G.; Kiermaier, A.; Bianchi, G.V.; Im, Y.H.; Pienkowski, T.; Liu, M.C.; Tseng, L.M.; Dowsett, M.; Zabaglo, L.; Kirk, S.; et al. Biomarker Analysis of the NeoSphere Study: Pertuzumab, Trastuzumab, and Docetaxel Versus Trastuzumab Plus Docetaxel, Pertuzumab Plus Trastuzumab, Or Pertuzumab Plus Docetaxel for the Neoadjuvant Treatment of HER2-Positive Breast Cancer. Breast Cancer Res. 2017, 19, 16. [CrossRef] [PubMed]

48. Holmes, F.A.; Espina, V.; Liotta, L.A.; Nagarwala, Y.M.; Danso, M.; McIntyre, K.J.; Osborne, C.R.; Anderson, T.; Krekow, L.; Blum, J.L.; et al. Pathologic Complete Response After Preoperative Anti-HER2 Therapy Correlates with Alterations in PTEN, FOXO, Phosphorylated Stat5, and Autophagy Protein Signaling. BMC Res. Notes 2013, 6, 507. [CrossRef]

49. Guarneri, V.; Dieci, M.V.; Frassoldati, A.; Maiorana, A.; Ficarra, G.; Bettelli, S.; Tagliafico, E.; Bicciato, S.; Generali, D.G.; Cagossi, K.; et al. Prospective Biomarker Analysis of the Randomized CHER-LOB Study Evaluating the Dual Anti-HER2 Treatment with Trastuzumab and Lapatinib Plus Chemotherapy as Neoadjuvant Therapy for HER2-Positive Breast Cancer. Oncologist 2015, 20, 1001-1010. [CrossRef]

50. Toomey, S.; Eustace, A.J.; Fay, J.; Sheehan, K.M.; Carr, A.; Milewska, M.; Madden, S.F.; Teiserskiene, A.; Kay, E.W.; O'Donovan, N.; et al. Impact of Somatic PI3K Pathway and ERBB Family Mutations on Pathological Complete Response (pCR) in HER2-Positive Breast Cancer Patients Who Received Neoadjuvant HER2-Targeted Therapies. Breast Cancer Res. 2017, 19, 87. [CrossRef]

51. Rimawi, M.F.; De Angelis, C.; Contreras, A.; Pareja, F.; Geyer, F.C.; Burke, K.A.; Herrera, S.; Wang, T.; Mayer, I.A.; Forero, A.; et al. Low PTEN Levels and PIK3CA Mutations Predict Resistance to Neoadjuvant Lapatinib and Trastuzumab without Chemotherapy in Patients with HER2 Over-Expressing Breast Cancer. Breast Cancer Res. Treat. 2018, 167, 731-740. [CrossRef]

52. Baselga, J.; Lewis Phillips, G.D.; Verma, S.; Ro, J.; Huober, J.; Guardino, A.E.; Samant, M.K.; Olsen, S.; de Haas, S.L.; Pegram, M.D. Relationship between Tumor Biomarkers and Efficacy in EMILIA, a Phase III Study of Trastuzumab Emtansine in HER2-Positive Metastatic Breast Cancer. Clin. Cancer Res. 2016, 22, 3755-3763. [CrossRef]

53. Perez, E.A.; de Haas, S.L.; Eiermann, W.; Barrios, C.H.; Toi, M.; Im, Y.H.; Conte, P.F.; Martin, M.; Pienkowski, T.; Pivot, X.B.; et al. Relationship between Tumor Biomarkers and Efficacy in MARIANNE, a Phase III Study of Trastuzumab Emtansine +/- Pertuzumab Versus Trastuzumab Plus Taxane in HER2-Positive Advanced Breast Cancer. BMC Cancer 2019, 19, 517.

54. Berns, K.; Horlings, H.M.; Hennessy, B.T.; Madiredjo, M.; Hijmans, E.M.; Beelen, K.; Linn, S.C.; Gonzalez-Angulo, A.M.; Stemke-Hale, K.; Hauptmann, M.; et al. A Functional Genetic Approach Identifies the PI3K Pathway as a Major Determinant of Trastuzumab Resistance in Breast Cancer. Cancer Cell 2007, 12, 395-402. [CrossRef] [PubMed]

55. Andre, F.; Hurvitz, S.; Fasolo, A.; Tseng, L.M.; Jerusalem, G.; Wilks, S.; O’Regan, R.; Isaacs, C.; Toi, M.; Burris, H.; et al. Molecular Alterations and Everolimus Efficacy in Human Epidermal Growth Factor Receptor 2-Overexpressing Metastatic Breast Cancers: Combined Exploratory Biomarker Analysis from BOLERO-1 and BOLERO-3. J. Clin. Oncol. 2016, 34, 2115-2124. [CrossRef] [PubMed]

56. Veeraraghavan, J.; De Angelis, C.; Reis-Filho, J.S.; Pascual, T.; Prat, A.; Rimawi, M.F.; Osborne, C.K.; Schiff, R. De-Escalation of Treatment in HER2-Positive Breast Cancer: Determinants of Response and Mechanisms of Resistance. Breast 2017, 34 (Suppl. S1), S19-S26. [CrossRef]

57. Pandolfi, P.P. Breast Cancer-Loss of PTEN Predicts Resistance to Treatment. N. Engl. J. Med. 2004, 351, 2337-2338. [CrossRef] [PubMed]

58. Crowder, R.J.; Lombardi, D.P.; Ellis, M.J. Successful Targeting of ErbB2 Receptors-is PTEN the Key? Cancer Cell 2004, 6, 103-104. [CrossRef] [PubMed]

59. Wang, L.; Zhang, Q.; Zhang, J.; Sun, S.; Guo, H.; Jia, Z.; Wang, B.; Shao, Z.; Wang, Z.; Hu, X. PI3K Pathway Activation Results in Low Efficacy of both Trastuzumab and Lapatinib. BMC Cancer 2011, 11, 248. [CrossRef] [PubMed] 
60. Loibl, S.; Majewski, I.; Guarneri, V.; Nekljudova, V.; Holmes, E.; Bria, E.; Denkert, C.; Schem, C.; Sotiriou, C.; Loi, S.; et al. PIK3CA Mutations are Associated with Reduced Pathological Complete Response Rates in Primary HER2-Positive Breast Cancer: Pooled Analysis of 967 Patients from Five Prospective Trials Investigating Lapatinib and Trastuzumab. Ann. Oncol. 2016, 27, 1519-1525. [CrossRef] [PubMed]

61. Barroso-Sousa, R.; Tyekucheva, S.; Pernas-Simon, S.; Exman, P.; Jain, E.; Garrido-Castro, A.C.; Files, J. PTEN alterations and Tumor Mutational Burden (TMB) as Potential Predictors of Resistance Or Response to Immune Checkpoint Inhibitors (ICI) in Metastatic Triple-Negative Breast Cancer (mTNBC). In Proceedings of the 2018 San Antonio Breast Cancer Symposium, Philadelphia (PA): AACR, San Antonio, TX, USA, 4-8 December 2018. Cancer Res. 2019, 79 (Suppl. S4), 5-12.

62. Mansour, W.Y.; Tennstedt, P.; Volquardsen, J.; Oing, C.; Kluth, M.; Hube-Magg, C.; Borgmann, K.; Simon, R.; Petersen, C.; Dikomey, E.; et al. Loss of PTEN-Assisted G2/M Checkpoint Impedes Homologous Recombination Repair and Enhances Radio-Curability and PARP Inhibitor Treatment Response in Prostate Cancer. Sci. Rep. 2018, 8, 3947. [CrossRef] [PubMed]

63. Mendes-Pereira, A.M.; Martin, S.A.; Brough, R.; McCarthy, A.; Taylor, J.R.; Kim, J.S.; Waldman, T.; Lord, C.J.; Ashworth, A. Synthetic Lethal Targeting of PTEN Mutant Cells with PARP Inhibitors. EMBO Mol. Med. 2009, 1, 315-322. [CrossRef] [PubMed]

(C) 2019 by the authors. Licensee MDPI, Basel, Switzerland. This article is an open access article distributed under the terms and conditions of the Creative Commons Attribution (CC BY) license (http://creativecommons.org/licenses/by/4.0/). 